\title{
An explicit formulation for the inverse transport problem using only external detectors - Part I: Computational Modelling
}

\author{
NANCY I. ALVAREZ ACEVEDO ${ }^{1 *}$, NILSON COSTA ROBERTY ${ }^{2}$ \\ and ANTÔNIO J. SILVA NETO ${ }^{1}$ \\ ${ }^{1}$ Department of Mechanical Engineering and Energy \\ Instituto Politécnico, IPRJ, Universidade do Estado do Rio de Janeiro, UERJ \\ P.O. Box 97282, 28601-970 Nova Friburgo, RJ, Brazil \\ ${ }^{2}$ Nuclear Engineering Program, PEN/COPPE \\ Universidade Federal do Rio de Janeiro, UFRJ \\ P.O. Box 68509, 21945-970 Rio de Janeiro, RJ, Brazil \\ E-mails: nacevedo@iprj.uerj.br / nilson@con.ufrj.br / ajsneto@iprj.uerj.br
}

\begin{abstract}
In the present work the inverse problem of identification of radiative properties, the total extinction and scattering coefficients, is analyzed and explicitly formulated based in an elementary semi-group theory. The Chandrasekhar discrete ordinates finite dimensional approximation of the angular variables is used for the direct problem representation with the stationary Linear Transport (Boltzmann) Equation in absorbing and scattering media in matrix form. For the inverse problem we suppose known the albedo operator from measured intensities at the boundaries of the medium. Here we analize the inverse problem for an $N$-dimensional medium and from the solution of the transmission problem we present an explicit form for the matrix that contains the total extinction and scattering coefficients.
\end{abstract}

Mathematical subject classification: 80A23, 15A29.

Key words: inverse problems, Discrete Ordinates Method, linear transport equation, radiative properties.

\#CAM-73/09. Received: 10/III/09. Accepted: 31/III/10.

*The first author is supported by FAPERJ. 


\section{Introduction}

The analysis of inverse radiative transfer and particle transport problems has several relevant applications in different areas such as reactor theory [5, 34]; heat transfer [40, 42, 47, 48]; remote sensing [1, 38, 59]; global warming models [23, 44]; natural waters radiative properties estimation [24, 31, 50, 57]; and tomography $[4,33,36]$; among many others.

In the last years advances in optical tomography have mainly been driven by applications in biomedical optics. Due to the existence of regions within the participating medium where the absorption coefficient is not much smaller than the scattering coefficient, or regions in which both are very low, both the diffusion approximation or the standard back propagation technique in X-ray tomography may fail $[4,37]$ the focus is directed to the construction of transport model-based image reconstruction, with a proper modelling of the absorption, scattering, and sometimes emission, phenomena using the radiative transfer equation and its equivalent Linear Transport (Boltzmann) equation.

Several methods have been developed for its solution, and more recently most attention has been devoted to the discrete ordinates method [6, 43, 46, 49, 56]; finite element method [40, 45, 60]; finite volume method [11, 25, 27], and Monte Carlo methods $[13,18,26]$.

Fiveland and Jessee $[21,22]$ tackled the direct radiative transfer problem in multidimensional media using a finite element formulation of the discrete ordinates methods.

Working with the problem of image reconstruction in two-dimensional media Reis and Roberty [54] proposed a domain partition consistent with a sourcedetector system for parallel beams of radiation. Carita Montero et al. [9] considered a similar problem for divergent beams of radiation.

Roberty and Silva Neto have studied with co-workers the inverse problem for radiative coefficients reconstruction $[3,8,28,55]$.

In the present work we introduce a new formalism for the radiative transfer and linear transport problem. Such formulation based in an elementary semi-group theory is very convenient for the treatment of the inverse problem in which we are seeking for estimates for the absorption and the scattering coefficients. 
To the best of our knowledge, the ideas exploited in this paper are new. The basic references for our development is a mix of fundamental references that comes from engineering [10, 19, 52]; from applied mathematics [7, 17, 29, 30, $32,35,39,51]$; and from physics [12, 20, 53].

\section{Mathematical formulation of the direct problem}

Consider an absorbing and scattering $N$ dimensional medium with no internal radiation sources, subjected to externally generated parallel beams of radiation. For the steady state situation, with no spectral dependency, the following mathematical formulation is obtained for the Linear Boltzmann equation [17, 29] which is usual in the modelling of the interaction of radiation with a participating medium,

$$
\begin{gathered}
\omega \cdot \nabla \phi(\omega, x)+\sigma_{t}(x) \phi(\omega, x) \\
=\int_{S^{N-1}} \sigma_{s}\left(x, \omega^{\prime} \cdot \omega\right) \phi\left(\omega^{\prime}, x\right) d \omega^{\prime} ;(\omega, x) \in S \times \Omega
\end{gathered}
$$

where $\phi \in L^{2}(S \times \Omega)$ is the constant velocity radiation intensity, $S^{N-1} \times \Omega$ is the domain of $\phi$ and $(x, \omega) \in \Omega \times S^{N-1}, S^{N-1} \cong[0,2 \pi)$ when $N=2$; $\sigma_{t} \in L^{\infty}(\Omega)$ is the total extinction coefficient (absorption + out scattering), $\sigma_{s}\left(x, \omega^{\prime} \cdot \omega\right)$ is the scattering coefficient.

Since $\omega^{\prime} \cdot \omega=\cos \theta_{0}$, with $\theta_{0}$ the angle between the direction of incident radiation $\omega^{\prime}$ and the emergent scattered radiation $\omega$, $\sigma_{s}(\cdot, s) \in L^{\infty}(\Omega)$, a.e. $s \in[-1,+1] ; \quad \sigma_{s}(x, \cdot) \in L^{1}([-1,+1])$, a.e. $x \in \Omega$.

In order to complete the mathematical formulation of the direct problem of radiative transfer in steady-state, i.e, to formulate the appropriate boundary value problem, we present next some definitions.

Let $\Gamma=\partial \Omega$ be the smooth boundary of the convex region $\Omega \subset R^{N}$ and $\Sigma^{ \pm}=\left\{(\omega, \sigma) \in S^{N-1} \times \Gamma ; \pm v(\sigma) \cdot \omega>0\right\}$ respectively the phase space surface of the incident and emergent radiation at the physical surface $\partial \Omega ; v$ represents the outward normal to $\partial \Omega$ and $\sigma$ a location at this boundary.

We also define the distance from the position $x$ to the boundary following directions $\pm \omega$ as

$$
\tau^{ \pm}(\omega, x)=\sup \{t \in R \mid x \pm t \omega \in \partial \Omega\}
$$


and

$$
\tau(\omega, x)=\tau^{+}(\omega, x)+\tau^{-}(\omega, x)
$$

as the effective thickness of the body $\Omega$ in the direction $\omega$ with support in $x$ and $L_{2}\left(\Sigma^{ \pm} ; \tau|v(\sigma) \cdot \omega| d \sigma d \omega\right)$ becomes the appropriate space for traces of the operators in boundary values problems related to equation (1). For a more detailed discussion see [16, 17, 29, 32, 35].

We are interested in two boundary value problems related to equation (1), respectively, the direct problem in which the incident radiation is prescribed in $L^{2}\left(\Sigma^{-} ; \tau|\nu(\sigma) \cdot \omega| d \sigma d x\right)$.

$$
\phi(\omega, \sigma)=\phi_{i n}(\omega, \sigma) \quad \text { for } \quad(\omega, \sigma) \in \Sigma^{-}
$$

and the adjoint problem for equation (1)

$$
\begin{gathered}
-\omega \cdot \nabla \phi^{*}(\omega, x)+\sigma_{t}(x) \phi^{*}(\omega, x) \\
=\int_{S^{N-1}} \sigma_{S}\left(x, \omega^{\prime} \cdot \omega\right) \phi^{*}\left(\omega^{\prime}, x\right) d \omega^{\prime} ;(\omega, x) \in S \times \Omega
\end{gathered}
$$

in which the emergent radiation

$$
\phi^{*}(\omega, \sigma)=\phi_{\text {out }}(\omega, \sigma) \quad \text { for } \quad(\omega, \sigma) \in \Sigma^{+}
$$

is prescribed in $L^{2}\left(\Sigma^{+} ; \tau|\nu(\sigma) \cdot \omega| d \sigma d x\right)$.

We obviously note that for each adjoint problem there is a direct problem such that

$$
\phi(-\omega, \sigma)=\phi^{*}(\omega, \sigma) \text { for } \quad(\omega, \sigma) \in \Sigma^{+}
$$

Observe that if $(\omega, \sigma) \in \Sigma^{+}$then $(-\omega, \sigma) \in \Sigma^{-}$, and these properties are a consequence of the adjointness of the scattering kernel in equation (1).

No internal sources are taken into account in our analysis, and the boundary of the medium is transparent, i.e., no reflections at the boundaries are considered. For a more general discussion about these questions see [17].

In general the transport theory is formulated in $L^{1}$, since the main physical quantity in this theory, the reaction rate, is in $L^{1}$ when the reaction coefficients are in $L^{\infty}$ and the radiation flux is in $L^{1}$. For our purpose of study, we will restrict our approach to $L^{2}$. More general results for $L^{p}, 1 \leq p<+\infty$, can be found in $[16,17,29]$. 
The steady state transport equation (1), is composed with the following operators:

The operator

$$
\begin{array}{rlc}
T_{0}: W_{2}\left(S^{N-1} \times \Omega\right) & \longrightarrow & L^{2}\left(S^{N-1} \times \Omega\right) \\
\phi(\omega, x) & \mapsto \quad\left[T_{0} \phi\right](\omega, x)=\omega \cdot \nabla \phi(\omega, x)
\end{array}
$$

where derivatives are in the sense of distributions.

The space $L^{2}\left(S^{N-1} \times \Omega\right)$ is the closure of $C\left(S^{N-1} \times \Omega\right)$ with respect to the norm

$$
\|\phi\|_{L^{2}}=\left(\int_{S^{N-1}} \int_{\Omega}|\phi(\omega, x)|^{2} d x d \omega\right)^{1 / 2}
$$

where $d \omega$ denotes the measure on $S^{N-1}$ associated with the Lebesgue measure in $\mathbf{R}^{N}$.

The space $W_{2}\left(S^{N-1} \times \Omega\right)$ is defined from $L^{2}\left(S^{N-1} \times \Omega\right)$ as

$$
W_{2}=\left\{\phi \in L^{2}\left(S^{N-1} \times \Omega\right) ; T_{0} \phi \in L^{2}\left(S^{N-1} \times \Omega\right)\right\},
$$

$W_{2}$ is a Hilbert space for the norm

$$
\|\phi\|_{L^{2}}=\left(\int_{S^{N-1}} \int_{\Omega}\left[|\phi(\omega, x)|^{2}+\left|T_{0} \phi(\omega, x)\right|^{2}\right] d x d \omega\right)^{1 / 2} .
$$

We note that

$$
L^{2}\left(S^{N-1} ; W^{1,2}(\Omega)\right) \subset W_{2} \subset L^{2}
$$

and $W_{2}$ is dense in $L^{2}$. The operator $T_{0}$ is accretive in $L^{2}$ and its maximal domain is $W_{2}$.

It is well known that $T_{0}$ is the generator of the strongly continuous group $\left[U_{0}(t) \phi\right](\omega, x)=\phi(\omega, x-t \omega)$ of isometries on $L^{2}\left(S^{N-1} \times \Omega\right)$ preserving the nonnegative functions, see $[16,17,29]$.

The second operator is

$$
\begin{array}{rlc}
A_{1}: L^{2}\left(S^{N-1} \times \Omega\right) & \longrightarrow & L^{2}\left(S^{N-1} \times \Omega\right) \\
\phi(\omega, x) & \mapsto \quad\left[A_{1} \phi\right](\omega, x)=\sigma_{t}(x) \phi(\omega, x)
\end{array}
$$

is a bounded operator which has continuous inverse for $\sigma_{t}(x)>0, x \in \Omega$. 
The composed operator $T_{1}=\left(T_{0}+A_{1}\right)$ is the generator of a strongly continuous group $U_{1}(t)=e^{t T_{1}}$.

$$
\left[U_{1}(t) \phi\right](\omega, x)=\exp \left[\int_{0}^{t} \sigma_{t}(x-s \omega) d s\right] \phi(\omega, x-t \omega) .
$$

The third operator

$$
\begin{aligned}
& A_{2}: L^{2}\left(S^{N-1} \times \Omega\right) \quad \longrightarrow \quad L^{2}\left(S^{N-1} \times \Omega\right) \\
& \phi(\omega, x) \quad \mapsto \quad\left[A_{2} \phi\right](\omega, x)
\end{aligned}
$$

where

$$
\left[A_{2} \phi\right](\omega, x)=-\int_{S^{N-1}} \sigma_{s}\left(x, \omega^{\prime} \cdot \omega\right) \phi\left(\omega^{\prime}, x\right) d \omega^{\prime},
$$

is compact.

The composed transport operator

$$
T=\left(T_{0}+A_{1}+A_{2}\right): W_{2}\left(S^{N-1} \times \Omega\right) \rightarrow L^{2}\left(S^{N-1} \times \Omega\right)
$$

generates a strongly continuous semigroup $U(t)=e^{-t T}$, for which does not exist an explicit formula.

\section{Formulation of the inverse problem with the Discrete Ordinates Method}

The inverse problem for external detectors and sources related to equation (1), can be stated in the following way:

We suppose the full knowledge of the incident boundary conditions

$$
\left\{\phi_{i n}(\omega, \sigma) ;(\omega, \sigma) \in \Sigma^{-}\right\}
$$

which is due to the control of external sources placed around the region $\Omega$ and also that the exit boundary conditions $\left\{\phi_{\text {out }}(\omega, \sigma) ;(\omega, \sigma) \in \Sigma^{+}\right\}$are known in part, being determined from external detector measurements.

We assume that we know the albedo operator $(\Lambda)$ in the case of transillumination of the region $\Omega$. The other possible situation is when the optical thickness of the medium is high, that is, the radiation is completely absorbed and scattered near its point of incidence on the surface. 
Following mathematical proofs of existence, uniqueness and stability of the inverse transport problem, see [58], we define the albedo operator as the influx to outflux mapping:

$$
\begin{aligned}
\Lambda: L^{2}\left(\Sigma^{-} ; \tau|v(\sigma) \cdot \omega| d \sigma d \omega\right) & \longrightarrow \quad L^{2}\left(\Sigma^{+} ; \tau|v(\sigma) \cdot \omega| d \sigma d \omega\right) \\
\phi_{\text {in }}(\omega, x) & \mapsto \quad\left[\left.\phi\right|_{\Sigma^{+}}\right](\omega, x)=\left[\left.\phi\right|_{\text {out }}\right](\omega, x)
\end{aligned}
$$

The inverse problem of determining the functions $\sigma_{t}$ and $\sigma_{s}$ from the knowledge of $\Lambda$ has been partially responded in Refs. [14] and [15] for the transient and steady state cases for dimension $\geq 2$, since in the one dimensional case the coefficients are expected to be reconstructed only for homogeneous media.

We here assume that the reconstruction is possible, that is, the albedo operator is injective and we treat only those problems related to the range and different orders of magnitude in the emergent flux (outflux).

The central hypotheses in this work lies in the relation between the albedo supposed to be characterized by its graph

$$
\left(\phi_{\text {in }}, \phi_{\text {out }}\right) \in L^{2}\left(\Sigma^{-} ; \tau|v(\sigma) \cdot \omega| d \sigma d \omega\right) \times L^{2}\left(\Sigma^{+} ; \tau|v(\sigma) \cdot \omega| d \sigma d \omega\right)
$$

and the bounded operator

$$
A_{1}+A_{2} \in \mathcal{L}\left(L^{2}\left(S^{N-1} \times \Omega\right)\right)
$$

In order to simplify the problem, we will use the Chandrasekhar discrete ordinates finite dimensional approximation of the angular variables [12]. This procedure will give a matrix formulation for the angular problem.

The Chandrasekhar discrete ordinates method uses either one dimensional or two dimensional quadrature formula to approximate the integral in the definition of the operator $A_{2}$.

For the one dimensional problem the quadrature uses the Legendre polynomials approximation for the scattering kernel and its weights are determined in such a way that the formula integrates correctly up to a polynomial of degree $2 M-1$. This generates the $S_{M}$ method (we use here $M$ instead the more usual subscript $N$ used in nuclear reactor physics terminology [39]) for the one dimensional transport equation. 
For multidimensional geometries there are different procedures for the determination of the quadratures sets and the corresponding weights. The reader can find appropriated information in Refs. [10, 19, 39].

In this way, the function with angular dependence, $\phi \in L^{2}\left(S^{N-1} \times \Omega\right)$ is replaced by the vector field $\phi_{(M)} \in L^{2}(\Omega)^{M}$, whose $M$ components are

$$
\phi_{(M) i}(x)=\phi\left(\omega_{i}, x\right), \quad i=1, \ldots, M .
$$

The operator $T_{0}$ is substituted in a straightforward manner by the following matrix operator:

$$
\begin{aligned}
T_{0}^{M}: L^{2}(\Omega)^{M} & \longrightarrow L^{2}(\Omega)^{M} \\
\phi_{M}(x) & \mapsto\left[T_{0}^{M} \phi_{M}\right](x)
\end{aligned}
$$

where $\left[T_{0}^{M} \phi_{M}\right](x)=\nabla \cdot\left(\Phi_{M}^{T} \mathcal{W}\right)$ and $\mathcal{W}$ is the matrix of vectors,

$$
\mathcal{W}=\left[\begin{array}{ccccc}
\vec{\omega}_{1} & 0 & 0 & \cdots & 0 \\
0 & \vec{\omega}_{2} & 0 & \cdots & 0 \\
\vdots & \vdots & \vdots & \cdots & 0 \\
0 & 0 & 0 & \cdots & \vec{\omega}_{M}
\end{array}\right]
$$

whose diagonal entries are the discrete directions $\vec{\omega}_{i}, i=1, \ldots, M$.

Note that $\vec{\omega}_{i}=\omega_{1 i} \vec{e}_{1}+\cdots+\omega_{M i} \vec{e}_{M}, i=1, \ldots, M$ are the cartesian representation of vectors $\vec{\omega}_{i}$. Obviously,

$$
\mathcal{W}=\mathcal{W}_{1} e_{1}+\cdots+\mathcal{W}_{M} e_{M}
$$

in a straightforward manner. The domain $\mathcal{D}\left(T_{0}^{M}\right)$ of the new operator will be the space $\prod_{m=1}^{M} H_{m, M}^{1}(\Omega)$, where

$$
H_{m, M}^{1}(\Omega)=\left\{\phi_{m} \in L^{2}(\Omega) \mid \omega_{m} \cdot \nabla \phi \in L^{2}(\Omega)\right\}
$$

The operators $A_{1}$ and $A_{2}$ are replaced, respectively, by

$$
\begin{aligned}
A_{1}^{M}: L^{2}(\Omega)^{M} & \longrightarrow L^{2}(\Omega)^{M} \\
\phi_{M} & \mapsto\left[\sigma_{t} \phi_{M}\right]
\end{aligned}
$$


and

$$
\begin{aligned}
A_{2}^{M}: L^{2}(\Omega)^{M} & \longrightarrow L^{2}(\Omega)^{M} \\
\phi_{M} & \mapsto\left[A_{2}^{M} \phi_{M}\right]
\end{aligned}
$$

Here, $\sigma_{t}^{M}=\sigma_{t} I^{M}$ is a diagonal matrix, with $I_{M}$ the identity matrix in $\mathbf{R}^{M \times M}$ and

$$
\left(\sigma_{s}^{M}\right)_{i j}=w\left(\omega_{i} \cdot \omega_{j}\right) \sigma_{s}\left(x, \omega_{i} \cdot \omega_{j}\right)
$$

is the $i, j$ entry of the scattering matrix, with $w$ the gaussian quadrature weight. Here the parameters in the two matrix $A_{1}^{M}$ and $A_{2}^{M}$ are in $L^{\infty}(\Omega)$.

The scattering matrix will present different types of symmetry, depending mainly on the dimension of the problem and on the geometry, but in all the situations, the matrix $A_{2}^{M}$ will be symmetric. To simplify the notation, we will continue to denote the finite dimensional matrix operators as $T_{0}, A_{1}$ and $A_{2}$.

The discrete ordinates form of equation (1) is

$$
\nabla \cdot\left[\phi^{T} \overrightarrow{\mathcal{W}}\right](x)=-\left[\phi^{T} A\right](x), \quad x \in \Omega
$$

where $A=A_{1}+A_{2}$.

In order to treat the boundary conditions for influx and outflux radiation, see equations (2) and (4), in the discrete ordinates form of the problem, we introduce the following definitions:

$$
\begin{aligned}
& \Gamma_{i}^{ \pm}(x)=\left\{\sigma \in \Gamma ; x \pm t \omega_{i}=\sigma\right\} \\
& \tau_{i}^{ \pm}(x)=\sup \left\{t \in \mathbf{R} \mid x \pm t \omega_{i} \in \partial \Omega\right\} \text { and } \\
& \tau_{i}(x)=\tau_{i}^{+}(x)+\tau_{i}^{-}(x) ;
\end{aligned}
$$

the physical meaning of the boundary space is the same as in section 2 .

Now $L^{2}\left(\Gamma_{i}^{ \pm} ; \tau_{i}|v(\sigma) \cdot \omega| d \omega\right)$ is the space for the component $i$ of the flux in the boundary. The signal \pm will be chosen accordingly to the angle between the outward normal $v(\sigma)$ and the direction $\omega_{i}$ and indicates whether the radiation is incident or emergent.

This situation generates a complicated angular range in the direct problem, because its boundary condition prescribes only the flux of incident radiation and the emergent flux must be determined in the same physical position in which the incident flux is prescribed, so the surface normal $v(\sigma)$ at each position 
$\sigma \in \Gamma$ divides $S^{N-1}$ in two ranges that are $\sigma$ dependent, and we can write $\phi$ as composed of two parts $\phi=\left[\phi_{-}, \phi_{+}\right]$, which contains the two types of information.

The same does occur in the inverse problem but with a fundamental difference: the albedo is supposed to be prescribed in such way that it determines the coefficients. Here we must answer a new question: What means in practice to know the albedo?

One possible answer is that in several experiments the emergent flux from different solutions of the transport equation for independent data at the incident boundary is measured by controlling the exit boundary through perfect detectors.

This situation corresponds to a generalization of the transmission tomography problem. So, we can suppose that we know the albedo through its graph in many boundary value measurements.

As stated before, the graph of the albedo operator is

$$
\left(\phi_{\text {in }}, \phi_{\text {out }}\right) \in L^{2}\left(\Sigma^{-} ; \tau|v(\sigma) \cdot \omega| d \sigma d \omega\right) \times L^{2}\left(\Sigma^{+} ; \tau|v(\sigma) \cdot \omega| d \sigma d \omega\right)
$$

but in a real experiment only a finite set of problems, that is, source-detector pairs will be determined.

The questions related to the instrumentation for this problem is out of the scope of this paper and will not be discussed here. Obviously, according to the type of the boundary $\Gamma=\partial \Omega$ for $\Omega$, this may become a very complicated problem. Meanwhile, assuming that this problem has been solved, we will build our model by using the discrete ordinates sets from the Discrete Ordinates Method.

Let $\left\{\phi_{i}(x) ; i=1, \ldots, M\right\}$ represent a set of experiments. We consider in the discrete problem given by equation (13) a set of $M$ fundamental boundary value problems generated by a linearly independent set of boundary conditions

$$
\left\{\phi_{i}(\sigma)=\left[\phi_{i}^{-}, \phi_{i}^{+}\right](\sigma)=\left[\phi_{\text {in }}, \phi_{\text {out }}\right](\sigma) ; i=1, \ldots, M, \sigma \in \Gamma\right\}
$$

where the positive range components have been determined by using the albedo operator.

Since the discrete problem given by equation (13) has a unique solution for a prescribed incident radiation, the set of $M$ vectors $\phi_{i}(x)$ are linearly indepen- 
dent and can be used to form the columns of a matrix $\Phi$, that is

$$
\Phi=\left[\begin{array}{cccc}
\mid & \mid & & \mid \\
\phi_{1} & \phi_{2} & \cdots & \phi_{M} \\
\mid & \mid & & \mid
\end{array}\right]
$$

which is prescribed on the boundary $\sigma \in \Gamma$ and denoted by $\Phi_{b}(\sigma)$.

These solutions of the problem given by equation (13) for the linearly independent set of boundary conditions can be grouped in a unique matrix equation

$$
\begin{aligned}
& \nabla \cdot\left[\Phi^{T} \overrightarrow{\mathcal{W}}\right](x)=-\left[\Phi^{T} A\right](x), \quad x \in \Omega \\
& \Phi(\sigma)=\Phi_{b}(\sigma), \quad \sigma \in \Gamma
\end{aligned}
$$

By construction, the matrix $\Phi(\sigma)$ has full rank, for each $\sigma \in \Gamma$. So

Lemma 3.1. If $\Phi(x)$ satisfies equation (13) and $\Phi(\sigma)=\Phi_{b}(\sigma)$ has full rank for every $\sigma \in \Gamma$, then $\Phi(x)$ has full rank for every $x \in \Omega$.

Proof. We know that for every column $\phi_{i}(\sigma)$ of $\Phi(\sigma), \sigma \in \Gamma$ the solution of equation (13) is unique, and that, by the linearity of the equation, if $\Phi(x)$ is a solution of (15) for the boundary data $\Phi_{b}(\sigma)$, then for every constant vector $c^{T} \in \mathbf{R}^{1 \times M}, c^{T} \Phi^{T}(x)$ satisfies equation (15) for the data $c^{T} \Phi^{T}(\sigma)$.

Suppose that there exists a $x \in \Omega$ such that $c^{T} \Phi^{T}(x)=0$. In such situation $\Phi_{c}^{T}=c^{T} \Phi^{T}$ is the unique solution of the problem given by equation (15) for the boundary data $\Phi_{c}(\sigma)=c^{T} \Phi(\sigma)$ which is by hypothesis $\neq 0$.

We face here a contradiction based on the uniqueness and on the nature of the solution for this kind of transport equation. In presence of scattering, every stationary non trivial solution can not have all angular components null inside the domain $\Omega$. So the contradiction establishes that no such point exists.

We can use this property to invert $\Phi$ and obtain for $\Phi \in C(\Omega)^{m \times M}$ that

$$
\nabla \cdot\left[\log \left(\Phi^{T}\right) \overrightarrow{\mathcal{W}}\right](x)=-A(x), x \in \Omega
$$

Equation (16) may be interpreted as a generalization of the transmission tomography equation since when we neglect the scattering, it can be integrated to produce the usual transmission tomography system for the discrete set of directions $\left\{\omega_{i}, i=1, \ldots, M\right\}$. 
In case of scattering these equations must be integrated in the whole domain $\Omega$ and by the divergence theorem the trace of the logarithm of matrix $\Phi^{T} \in$ $C(\Omega)^{M \times M}$ is related with the matrix of coefficients $A$, that is

$$
\int_{\Gamma} \log \left(\Phi^{T}\right) \overrightarrow{\mathcal{W}} \cdot v(\sigma) d \sigma=-\int_{\Omega} A(x) d x
$$

These last equations give an explicit inversion formula for the operator $A$. Depending on the dimension of the domain, that is, one, two or three dimensions, we will define different strategies for the implementation of the problem of coefficients reconstruction, all using equations (16) and (17).

For a one-dimensional homogeneous media with slab geometry and boundaries in $x=0$ and $x=\tau_{1}$ we obtain:

$$
A=-T \ln \left[\Phi\left(\tau_{1}\right) \Phi^{-1}(0)\right] \frac{1}{t}
$$

where $t$ is the slab thickness, $T$ corresponds to $T_{0}$, and $\Phi(0)$ and $\Phi\left(\tau_{1}\right)$ the incident and emergent radiative flux in the boundaries at $x=0$ and $x=\tau_{1}$, respectively.

As an example of the new methodology application we present the study of the one-dimensional stationary problem in an accompanying article [2].

\section{Conclusions}

In this article we derived a new explicit formulation for an inverse radiative transfer problem for identification of the extinction and scattering coefficients in an $N$-dimensional participating media using only external detectors.

Here we analyze the albedo operator and present an explicit expression for the matrix $A$ that contains the total extinction and scattering coefficients.

In an accompanying article [2] we apply this formulation for a one-dimensional inverse radiative transfer problem. We will show the derivation of the explicit equation for matrix $A$ and the strategy developed to extract the total extinction and scattering coefficients contained in it from albedo operator information.

Acknowledgements. The authors acknowledge the financial support provided by CNPq, Conselho Nacional de Desenvolvimento Científico e Tecnológico, and 
FAPERJ, Fundação Carlos Chagas Filho de Amparo à Pesquisa do Estado do Rio de Janeiro.

\section{REFERENCES}

[1] F. Ahmad, S. Oppenheimer and M. Razzaghi, A discrete bidirectional reflectance model in remote sensing. J. Quant. Spectrosc. Radiat. Transfer, 77 (2003), 335-343.

[2] N.I. Alvarez Acevedo, N.C. Roberty and A.J. Silva Neto, An explicit formulation for the inverse transport problem using only external detectors. Part II: Application to onedimensional homogeneous and heterogeneous participating media. Comput. Appl. Math (2010).

[3] N.I. Alvarez Acevedo, N.C. Roberty and A.J. Silva Neto, One dimensional inverse radiative transfer problem with time-dependent boundary conditions. $4^{\text {th }}$ International Conference on Inverse Problems in Engineering: Theory and Practice, Proc., Angra dos Reis, Brazil (2002).

[4] S.R. Arridge Optical tomography in medical imaging. Inverse Prob., 15 (1999), R41-R93.

[5] M. Aydin and M.A. Atalay, Inverse neutron diffusion problems in reactor design, J. Nucl. Sci. Technol., 44(9) (2007), 1142-1148.

[6] L.B. Barichello, P. Rodriguez and C. Siewert, An analytical discrete-ordinates solution for dual-mode heat transfer in a cylinder. J. Quant. Spectrosc. Radiat. Transfer, 73(2002), 583-602.

[7] A.N. Bondarenko, The structure of the fundamental solution of the time-independent transport equation. J. Math. Anal Appl., 221(2) (1998), 430-451.

[8] R.F. Carita Montero, N.C. Roberty and A.J. Silva Neto, Reconstruction of absorption and scattering coefficients with a discrete ordinates method consistent with the source-detector system. 4th International Conference on Inverse Problem in Engineering: Theory and Practice, Proc., Angra dos Reis, Brazil (2002).

[9] R.F. Carita Montero, N.C. Roberty and A.J. Silva Neto, Absorption coefficient estimation in heterogeneous media using a domain partition consistent with divergent beams. Inverse Prob. Eng., 9 (2001), 587-617.

[10] K.M. Case and P.F. Zweifel, Linear Transport Theory. Addison Weslley, New York (1967).

[11] J.C. Chai. P-f. Hsu and Y.C. Lam, Three-dimensional transient radiative transfer modeling using the finite-volume method. J. Quant. Spectrosc. Radiat. Transfer, 86(3) (2004), 299-313.

[12] S. Chandrasekhar, Radiative Transfer. Dover Publishing, Inc., New York (1960).

[13] Y. Chen and K.N. Liou, A Monte Carlo method for 3D thermal infrared radiative transfer. J. Quant. Spectrosc. Radiat. Transfer, 101(1) (2006), 166-178.

[14] M. Choulli and P. Stefanov, An inverse boundary value problem for the stationary transport equation. Osaka J. Math., 36(1) (1998), 87-104. 
[15] M. Choulli and P. Stefanov, Inverse scattering and inverse boundary value problem for the linear Boltzmann equation. Commun. Part. Diff. Eq., Taylor and Francis, 21(5-6) (1996), $763-785$.

[16] R. Cipollati, An introduction to the problems of parameters estimation in partial differential equations. $57^{\circ}$ Seminário Brasileiro de Análise, Viçosa, MG, Brasil (2003). (In Portuguese).

[17] R. Dautray and J.-L. Lions, Evolutions problems II. Mathematical Analysis and Numerical Methods for Sciences and Technology, Springer Verlag, Berlin, Heidelberg, 6(1993).

[18] G. Demirkaya, F. Arinç, N. Selçuk and I. Ayranci, Comparison between performances of Monte Carlo method and method of lines solution of discrete ordinates method. J. Quant. Spectrosc. Radiat. Transfer, 93(1-3) (2005), 115-124.

[19] J.J. Duderstadt and W.R. Martin, Transport Theory. John Wiley and Sons, Inc., USA (1979).

[20] H. Emamirad, Scattering theory for linearized Boltzmann equation. Survey and news results. Transport Theor. Stat., 16(4-6) (1987), 4503-4528.

[21] W.A. Fiveland and J.P. Jessee, Comparison of discrete-ordinates formulation for radiative heat transfer in multidimensional geometries. J. Thermophys. Heat Transfer, 9(1) (1995), $47-54$.

[22] W.A. Fiveland and J.P. Jessee, Finite element formulation of the discrete-ordinates methods for multidimensional geometries. J. Thermophys. Heat Transfer, 8(3) (1994), 426-433.

[23] S. K. Foong, An accurate analytical solution of a zero-dimensional greenhouse model for global warmingsource. Eur. J. Phys., 27 (2006), 933-942.

[24] A.H. Hakim and N.J. McCormick, Ocean optics estimation for absorption, backscattering, and phase function parameters. Appl. Opt., 42(6) (2003), 931-938.

[25] P. Hassanzadeh and G.D. Raithby, Finite-volume solution of the second-order radiative transfer equation: Accuracy and solution cost. Numer. Heat Transfer Part B, 53(4) (2008), 374-382.

[26] H. Iwabuchi, Efficient Monte Carlo methods for radiative transfer modeling. J. Atmos. Sci., 63(9) (2006), 2324-2339.

[27] H. Jinbo, R. Liming and T. Heping, Effect of anisotropic scattering on radiative heat transfer in two-dimensional rectangular media. J. Quant. Spectrosc. Radiat. Transfer, 78 (2003), $151-161$.

[28] A.T. Kauati, A.J. Silva Neto and N.C. Roberty, A Source-detector methodology for the construction and solution of the one-dimensional inverse transport equation. Inverse Prob. Eng., 9 (2001), 45-66.

[29] H.G. Kaper, C.G. Lekkerkjer and J. Hejtmanek, Spectral methods in linear transport theory. Oper. Theory Adv. Appl., Birkhäuser, Basel, 5 (1982).

[30] T. Kato, Perturbation theory of linear operators. Classics Math., Springer-Verlag, Berlin (1995). 
[31] A. Kaşkaş, C. Tezcan and M.Ç. Güeçyüz, An application of transport theory for optical oceanography: the isotropic point source. J. Opt. A: Pure Appl. Opt., 8(8) (2006), 683-688.

[32] O. Kavian, Four lectures on parameter identification in elliptic partial differential equations. Three courses on Partial Differential Equations, IRMA Lect. Math. Theor. Phys., de Gruyter(ed.), Berlin, 4 (2003), 125-162.

[33] F.E. Khettabi and M.A. Hussein, An inverse problem for three-dimensional $x$-ray scatter/Transmission imaging. Inverse Probl., 19 (2003), 477-495.

[34] V.G. Kinelev, P.M. Shkapov and V.D. Sulimov, Application of global optimization to VVER-1000 reactor diagnostics. Prog. Nucl. Energ., 43(1-4) (2003), 51-56.

[35] A. Kirsch, An introduction to the mathematical theory of inverse problems. Appl. Math. Sci., Springer Verlag, Berlin, 120 (1996).

[36] A.D. Klose and A.H. Hielscher, Optical tomography using the time-independent equation of radiative transfer-Part 2: Inverse model. J. Quant. Spectrosc. Radiat. Transfer, 72 (2002), 715-732.

[37] A.D. Klose, U. Netz, J. Beuthan and A.H. Hielscher, Optical tomography using the timeindependent equation of radiative transfer - Part 1: Forward model. J. Quant. Spectrosc. Radiat. Transfer, 72 (2002), 691-713.

[38] B. Koetz, F. Baret, H. Poilvé and J. Hill, Use of coupled canopy structure dynamic and radiative transfer models to estimate biophysical canopy characteristics. Remote Sens. Environ., 95(1) (2005), 115-124.

[39] E.E. Lewis and W.F. Miller, Jr., Computational Methods of Neutron Transport. John Wiley and Sons, Inc. USA (1984).

[40] L.H. Liu and B.X. Li, Inverse radiation problem of axisymmetric turbulent sooting free flame. J. Quant. Spectrosc. Radiat. Transfer, 75 (2002), 481-491.

[41] L.H.Liu and L.J. Liu, Discontinuous finite element approach for transient radiative transfer equation. J. Heat Trans., 129(8) (2007), 1069-1074.

[42] L.H. Liu and G.L. Man, Reconstruction of time-averaged temperature of non-axisymmetric turbulent unconfined sooting flame by inverse radiation analysis. J. Quant. Spectrosc. Radiat. Transfer, 78 (2003), 139-149.

[43] L.H. Liu and L.M. Ruan, Numerical approach for reflections and transmittance of finite plane-parallel absorbing and scattering medium subjected to a normal and diffuse incidence. J. Quant. Spectrosc. Radiat. Transfer, 75 (2002), 637-646.

[44] J. Liu, X. Xia, P. Wang, Z. Li, Y. Zheng, M. Cribb and H. Chen. Significant aerosol direct radiative effects during a pollution episode in northern China. Geophys. Res. Lett., 34(23) (2007), L23808.

[45] T.A. Manteuffel and K.J. Ressel, Least-squares finite-element solution of the neutron transport equation in diffusive regimes. SIAM J. Numer. Anal., 35(2) (2002), 806-835.

[46] V.A. Markel, G.Y.Panasyuk and J.C. Schotland, New approach to solution the radiative 
transport equation. Biomedical Optics 2006 Technical Digest, (CD) OSA, Washington DC. (2006).

[47] M.P. Mengüç and P. Dutta, Scattering tomography and its application to sooting diffusion flames. J. Heat Transfer, 116 (1994), 144-151.

[48] A. Milandri, F. Asllanaj and G. Jeandel, Determination of radiative properties of fibrous media by an inverse method-comparison with the Mie Theory. J. Quant. Spectrosc. Radiat. Transfer, 74 (2002), 637-653.

[49] C. Muresan, R. Vaillon, Ch. Menezo and R. Morlot, Discrete ordinates solution of coupled conductive radiative heat transfer in a two-layer slab with Fresnel interfaces subject to diffuse and obliquelly collimated irradiation. J. Quant. Spectrosc. Radiat. Transfer, 84 (2004), 551-562.

[50] A.H. Nasr, B.M. El Leithy and A.K. Helmy, Assessment of some water quality parameters using MODIS data along the Red Sea Coast, Egypt. ICGST Int. J. on Graphics, Vision and Image Processing, GVIP 7(3) (2007), 25-30.

[51] F. Natterer, The Mathematics of Computarised Tomography. John Wiley and Sons, Inc., New York (1986).

[52] M.N. Özisik, Radiative Transfer and Interactions with Conduction and Convection. John Wiley and Sons, Inc., USA. (1973).

[53] M. Reed and B. Simon, Scattering theory. Methods of Modern Mathematical Physics, Academic Press, New York, 3 (1979).

[54] M.L. Reis and N.C. Roberty, Maximum entropy algorithms for image reconstruction from projections. Inverse Prob., 8 (1992), 623-644.

[55] N.C. Roberty, On the influx to the outflux mapping to the transport equation. $4^{\text {th }}$ International Conference on Inverse Problems in Engineering: Theory and Practice, Proc., Angra dos Reis, Brazil (2002).

[56] C. Siewert, A discrete-ordinates solution for radiative-transfer models that include polarization effects. J. Quant. Spectrosc. Radiat. Transfer, 64 (2000), 227-254.

[57] R.P. Souto, H.F. Campos Velho and S. Stephany, Reconstruction of vertical profiles of the absorption and scattering coefficients from multispectral radiance. Math. Comput. Simulat., Elsevier Science Publ., Amsterdam, 73(1) (2006), 255-267.

[58] P. Stefanov, Inverse problem in transport theory. Inside Out: Inverse Problems and Applications, MSRI Publications, 47 (2003).

[59] L.L. Strow, S.E. Hannon, S. de Souza-Machado, H.E. Motteler and D. Tobin, An overview of the AIRS radiative transfer model. IEEE Trans. Geosci. Remote Sens., 41(2) (2003), 303-343.

[60] T. Tarvainen, M. Vauhkonen, V. Kolehmainen and J.P. Kaipio, Finite-element approximations for the radiative transfer equation. Int. J. Numer. Meth. Eng., 65(3) (2005), $383-405$. 